Standard 3: 13/14 (93\%) patients were commenced on Ambisome® if they had $>96$ hours of neutropenic fever.

Standard 4: 2/2 (100\%) patients were initiated on salvage therapy if there was further progression of fungal disease.

Standard 5: $2 / 2(100 \%)$ patients were continued on salvage therapy if there was incomplete resolution on the HRCT.

Standard 6: 7/8 (88\%) patients were commenced on voriconazole following complete resolution on the HRCT.

Following initiation with prophylactic itraconazole (Standard 2) 6 of 24 patients were switched to prophylactic Ambisome ${ }^{\circledR}$ due to deranged liver function tests.

Following initiation of treatment dose Ambisome ${ }^{\circledR}$ (Standard 3) 4 of 14 patients were switched to treatment dose caspofungin due to adverse effects of Ambisome ${ }^{\circledR}$ treatment: lip swelling, nephrotoxicity or chest pain.

Switching to Ambisome ${ }^{\circledR}$ for standard 2 and to caspofungin for standard 3 was beyond the scope of the SOP as there is no information on what alternative agents should be used should the patient experience side effects with the original agents.

Conclusion The audit has shown that the current SOP needs to include where and what agents should be used should deviation from standard protocol become necessary such as when patients are unable to tolerate certain medication due to contra-indications or side effects.

\section{P 26 AN AUDIT EVALUATING ADHERENCE TO ANTI-FUNGAL THERAPY IN PAEDIATRIC STEM CELL TRANSPLANT (SCT) PATIENTS}

Poonam Lumb. Imperial College Healthcare NHS Trust

10.1136/archdischild-2015-308634.34

Aim To evaluate adherence to anti-fungal therapy in accordance with 'The Invasive Fungal Infections: Investigation and Management' ${ }^{1}$ Standard Operating Procedure (SOP) in Stem Cell Transplant (SCT) paediatric patients.

Method Paediatric patients prescribed anti-fungal therapy from April 2013 onward were identified. Medical notes were analysed retrospectively to determine if the SOP had been adhered to. Data were recorded on a piloted data collection tool. All paediatric SCT patients admitted and treated at the hospital from Apr 2013 to Feb 2014 were included.

Audit Standards 100\% of patients should be:

1. Treated in accordance with the guidance stated in the SOP.

2. Commenced on prophylactic itraconazole.

3. Commenced on Ambisome ${ }^{\circledR}$ treatment if they have $>96$ hours of neutropenic fever.

4. Initiated on salvage therapy for further progression of invasive fungal disease.

5. Continued on salvage therapy if there is some but not complete resolution following a high resolution CT scan (HRCT).

6. Commenced on voriconazole secondary prophylaxis if there is complete resolution following a HRCT.

Results A total of 25 patients were identified for inclusion; one of these had to be excluded as the medical notes could not be tracked.

Standard 1: 22/24 (92\%) patients were treated in accordance to the guidance set in the SOP. One was initiated on treatment voriconazole instead of Ambisome ${ }^{\circledR}$ (standard 3) and another was initiated on secondary prophylaxis itraconazole instead of voriconazole (standard 6).

Standard 2: 24/24 (100\%) patients were commenced on prophylactic itraconazole.

\section{REFERENCE}

1 NHS Trust. Invasive Fungal Infections: Investigation and Management Standard Operating Procedure. 2013,1-12. 